\title{
Controlling self-assembled structure of Au nanoparticles by convective self-assembly with liquid-level manipulation
}

\section{$\operatorname{AUTHOR}(\mathrm{S}):$}

Hanafusa, Tatsuya; Mino, Yasushi; Watanabe, Satoshi; Miyahara, Minoru T.

\section{CITATION:}

Hanafusa, Tatsuya ... [et al]. Controlling self-assembled structure of Au nanoparticles by convective self-assembly with liquid-level manipulation. Advanced Powder Technology 2014, 25(2): 811-815

\section{ISSUE DATE:}

2014-03

URL:

http://hdl.handle.net/2433/187112

\section{RIGHT:}

(c) 2014 The Society of Powder Technology Japan. Published by Elsevier B.V.; This is not the published version. Please cite only the published version.; この論文は出版社版でありません。引用の際には出版社版を ご確認ご利用ください。 


\section{Controlling Self-Assembled Structure of $\mathrm{Au}$}

\section{Nanoparticles by Convective Self-Assembly with}

\section{Liquid-Level Manipulation}

Tatsuya Hanafusa, Yasushi Mino, Satoshi Watanabe, and Minoru T. Miyahara*

Department of Chemical Engineering, Kyoto University,

Katsura, Nishikyo, Kyoto 615-8510, Japan

* Corresponding author

miyahara@cheme.kyoto-u.ac.jp 


\begin{abstract}
Microwire networks composed of noble metal particles are promising for the use of transparent conductive films. Bottom-up approaches can offer a route to establishing a fabrication technique that is robust and cost-effective, and template-assisted self-assembly techniques are widely used. However, they require additional processes to prepare templates and generally suffer from the difficulty in a large-scale fabrication. A template-free technique thus waits to be developed.

In the present study, we explore a template free technique to fabricate colloidal networks of $\mathrm{Au}$ nanoparticles. We combine the convective self-assembly method with a liquid-level manipulation scheme in which the suspension is periodically pumped out. By using the technique, we successfully fabricate stripe, grid, and triangle patterns with controlled periodicity and examine the relationship between operation parameters and the resultant structures. We then measure the transparency and conductivity of a grid pattern to demonstrate the property as the transparent conductive film.
\end{abstract}

Keywords: Self-organization, Au network pattern, Convective self-assembly, Template-free fabrication, Transparent conductive film 


\section{Introduction}

Noble metal nanoparticles have attracted much attention because of their size-dependent catalytic, photonic, electronic, and magnetic properties. Their collective structures with high regularity are also attractive because they show unique photonic and chemical characteristics and can be used for various applications as photonic crystals [1-3], chemical sensors [4], and surface-enhanced Raman scattering (SERS) substrates [5]. The present study focuses on the formation of network patterned structures of metal nanoparticles on a substrate, which are promising candidates as transparent conductive materials. The conductivity of these network structures is dependent on the particulate lines and the transparency depends on the spacing [6]. Several bottom-up techniques have been proposed to produce patterned structures of particles, among which template-assisted self-assembly is popular [7-10]. The template-assisted approaches enable facile fabrication of patterned structures with high precision, although a different template is required for making any changes in the pattern. This is a major issue because templates are created by costly top-down lithography techniques. Hence, a template-free self-assembly technique is strongly required.

We have studied the vertical deposition convective self-assembly (CSA) [11] in which particles are assembled toward the meniscus tip by an evaporation-induced upward convection and are deposited at the meniscus tip as a close-packed array due to the lateral capillary forces acting between the particles. This technique was originally developed to form uniform particulate films; however, we have found that under specific conditions, the method can produce stripe patterns composed of colloidal particles [12]. In addition, we have shown that repeating the stripe formation after rotating the substrate by $90^{\circ}$ results in a grid pattern [13]. This demonstrates the potential of this template-free pattern formation technique. However, the limitation of this technique lies in the lack of control of the stripe periodicity because the stripe width and spacing are predominantly determined by the particle 
concentration. We have solved this problem and controlled the stripe width and spacing by incorporating liquid-level manipulation (LLM) into the CSA process, by a technique we term CSA-LLM [14].

In the present study, we fabricate patterns of stripe, grid, and triangle structures of gold nanoparticles by the CSA-LLM technique. We investigate the relationship between the periodicity of the resultant structures and the operation parameters under fixed conditions of particle concentration and temperature. From our results we demonstrate that our technique can produce predetermined particulate line widths and spacings.

\section{Experimental Section}

Gold nanoparticles with a diameter of $20 \mathrm{~nm}$ (Finesphere gold W101) with surface adsorbed comb-shaped block polymeric protective agents were purchased from Nippon Paint Co., Ltd. (Japan). The suspensions were diluted to a desired concentration with ultrapure water (resistivity $>18 \mathrm{M} \Omega \bullet \mathrm{cm}$ ). The volume fraction of particles $\phi$ was fixed to be $1.0 \times 10^{-4}$ vol/vol in most of the experiments unless otherwise stated. Cover glasses with dimensions of $18 \times 18 \mathrm{~mm}^{2}$ (Matsunami Glass Ind., Ltd., Japan) were used as substrates. The substrates were treated with a plasma cleaner (PDC-32G, Harrick Plasma Inc., USA) after a preliminary cleaning procedure in which they were washed in an ultrasonic bath for at least 5 minutes with acetone, ethanol, and ultrapure water.

Figure 1 is the schematic of the experimental setup. A glass substrate was vertically immersed in a suspension of gold nanoparticles and placed in an incubator (IS600, Yamato Scientific Co., Ltd., Japan) under a controlled temperature of $70{ }^{\circ} \mathrm{C}$. The humidity in the incubator was measured to be less than $10 \%$ during the assembly experiments, although we did not attempt to control the humidity. The particles in the suspension were transported upward by convection induced by solvent evaporation and were deposited at the meniscus tip. 
After a specific duration, the suspension was pumped out of the reservoir by a programmable syringe pump (Nexus 3000, Chemyx Inc., USA) to decrease the liquid level. When the syringe pump was paused, particles assembled at the meniscus tip to form a particulate line. Due to the suction of the suspension, after a certain period of time (i.e., the time period required for particle deposition $t_{\mathrm{p}}[\mathrm{s}]$ ), the liquid level quickly decreased (to a liquid-level drop distance $\left.l_{\mathrm{p}}[\mu \mathrm{m}]\right)$. A stripe pattern could be obtained by repeating the particle deposition and suction. We varied $t_{\mathrm{p}}$ from 7 to $60 \mathrm{~s}$ and $l_{\mathrm{p}}$ from 67 to $401 \mu \mathrm{m}$. The rate of drop of the liquid level was set at $100 \mu \mathrm{m} / \mathrm{s}$ in the present study. The dropping rate and distance of the liquid level was calculated by dividing the suction rate and amount, respectively, by the surface area of the suspension in the beaker.

After the particle assembly, the samples were characterized with a digital microscope (VHX-VK-600, Keyence Corp., Japan) and an atomic force microscope (AFM; SPM-9700, SHIMADZU Corp., Japan). The sheet resistance and transmittance of the grid patterns were measured with a four-pin probe resistivity meter (Loresta-EP MCP-T360, Mitsubishi Chemical Analytech Co., Japan) and a fiber optic spectrometer (USB4000, Ocean Optics, Inc., USA), respectively.

\section{Results and Discussion}

\subsection{Structure control by CSA-LLM}

Figure $2 \mathrm{a}$ shows the typical example of a stripe pattern fabricated by the CSA-LLM technique in which the $t_{\mathrm{p}}$ and $l_{\mathrm{p}}$ were set to be $7 \mathrm{~s}$ and $107 \mu \mathrm{m}$, respectively. The white lines are stripes composed of gold nanoparticles, while the dark bands indicate the spacing where almost no particles are deposited. The average width of the stripe and the spacing between the stripes are measured to be $13 \mu \mathrm{m}$ and $113 \mu \mathrm{m}$, respectively. The stripe pattern uniformly covered the whole substrate of several $\mathrm{cm}^{2}$. The AFM image of the stripe shown in Figure $2 \mathrm{~b}$ indicates a 
bilayer construction with winding particulate lines vertically extended from the top portion of the stripe. It should be noted that such winding lines are unique to the gold particles we used in the present study. The structures assembled from citrate-protected gold particles and silica particles show a clear edge at the top portion of the stripes. This can thus be attributed to the fingering instability [15] of the thin liquid film containing the protecting polymer on the gold particle surface, as indicated in the experimental section. Variation of the $t_{\mathrm{p}}$ and $l_{\mathrm{p}}$ changed the periodicity of the stripe patterns without disturbing the regularity of the structure, as shown in Figure 3. At a constant particle concentration and temperature, a higher values of $t_{\mathrm{p}}$ yielded wider stripes (Figure 3a), while smaller values of $t_{\mathrm{p}}$ resulted in narrower stripes (Figure $3 \mathrm{c}$ ). Similarly, higher values of $l_{\mathrm{p}}$ produced wider spacing. The resultant stripes were confirmed to consist of bilayer structures under the conditions employed in the present study.

Figure 4 shows the relationship between the spacing obtained and $l_{\mathrm{p}}$. The spacing obtained showed a linear relationship (with a slope of unity) with $l_{\mathrm{p}}$. This relationship, which indicates that the spacing is almost equal to $l_{\mathrm{p}}$, seems natural; however, it should be noted that this is a simplified form of the relationship obtained in our previous study (Eq. (3) in Ref. 14). The spacing can depend on the $t_{\mathrm{p}}$ because the solvent evaporation gradually lowers the liquid level before the liquid-level drop occurs by aspiration. The simple relationship presented in Figure 4 is hence applicable for small values of $t_{\mathrm{p}}$ where the liquid drop by the evaporation is negligible. This relationship is also applicable for high concentration conditions (typically $\phi \geq$ $10^{-4}$ ) in which the growth rate of a particulate line can be assumed to be the same as that of the evaporation, which eliminates the contribution of evaporation, resulting in a simple relationship. The coefficient of variance $(\mathrm{CV})$ of the spacings obtained in samples prepared by CSA-LLM was found to be approximately $9 \%$, which is better than that observed for spacings obtained in samples by the normal CSA technique without LLM (11\%). This demonstrates the high performance of CSA-LLM in the creation of spacings in a controlled manner. The 
variation in the spacing could result from the non-uniform wettability of the substrate surface and the accuracy of the syringe pump, which can be improved.

The stripe width linearly increased with $t_{\mathrm{p}}$, as seen in Figure 5 , because higher values of $t_{\mathrm{p}}$ allow a larger number of particles to deposit at the meniscus tip. Further, the CV of the stripe widths in Figure 5 is $6 \%$, which is much less than $18 \%$ observed in the case of normal CSA without LLM. This result demonstrates that the stripe width can be precisely controlled by changing the deposition period $t_{\mathrm{p}}$ by CSA-LLM. It is noteworthy that the linear fit of the data in Figure 5 shows a $y$-intercept at $t_{\mathrm{p}}=0 \mathrm{~s}$ because the slope, which expresses the growth rate of a stripe, varies inversely with the layer number of the stripe. In the early stages of the stripe formation, monolayer stripes would form and grow proportionally with time. Subsequently, at a certain $t_{\mathrm{p}}$, bilayer stripes begin to grow with half the growth rate of the monolayers. The data in Figure 5 corresponds to the bilayer formation.

In our previous study [14], we demonstrated that the model of particulate continuous film formation [11] is applicable to stripe formation by CSA-LLM. Here, we obtain an equation to express the volume of a stripe instead of the stripe width. Because the particle flux from a suspension is determined by the evaporation rate at the drying region around the meniscus tip, as shown in Figure 6, a simple mass balance around the meniscus tip region gives the following equation to express the volume of a stripe $V_{\mathrm{s}}$ :

$$
V_{\mathrm{s}}=J_{\mathrm{e}} L W_{\mathrm{s}} \frac{\phi}{1-\phi} t_{\mathrm{p}}
$$

In the above equation, $J_{\mathrm{e}}$ is the rate of solvent evaporation per unit of drying length at the meniscus tip, $L$ is the drying length, and $W_{\mathrm{s}}$ is the width of a substrate. In this equation, $\phi$ and $t_{\mathrm{p}}$ are operating parameters and $W_{\mathrm{s}}$ is a constant, which are all known. The value of $J_{\mathrm{e}}$ along the drying length is not necessarily the same as that of a bulk suspension because the heat transfer from the substrate increases $J_{\mathrm{e}}$. For a thin water film spread on a substrate, in which the particulate film formed at the meniscus tip is neglected for simplicity, we obtained $J_{\mathrm{e}}$ at 70 
${ }^{\circ} \mathrm{C}$ as $3.5 \times 10^{-6} \mathrm{~m}^{3} / \mathrm{m}^{2} \cdot \mathrm{s}$ by calculating a wet-bulb temperature by including the effects of heat transfer from the substrate and air with a boundary fluid thickness of $1.5 \mathrm{~mm}$. By using these values, Eq. (1) can predict the stripe volume at an arbitrary value of $t_{\mathrm{p}}$ for a given $L$. To verify the quantitative validity of the model, we converted the measured stripe width into the stripe volume $V_{\mathrm{s}}$ by measuring the stripe thickness by AFM. Figure 7 shows the relationship between $V_{\mathrm{s}}$ and $t_{\mathrm{p}}$ for three different particle concentrations. The stripe volume increases proportionally with time and the slope is larger for a larger particle concentration. Figure 7 also includes the results of the calculations obtained from Eq. (1). In the calculation, since the drying length $L$ is not theoretically obtainable, we fitted the data for $\phi=1.0 \times 10^{-4}$ (which is represented by the broken line in Figure 7) and obtained the value of $L$ to be $144 \mu \mathrm{m}$ from the slope of the broken line. We applied Eq. (1) to different concentration conditions using the obtained value of $L$ with the assumption that the drying length is constant under fixed temperature conditions. As seen in Figure 7, the results of the calculation (solid lines) agreed fairly well with the results of the experiments, demonstrating that the resultant stripe volume can be predicted by using Eq. (1).

\subsection{Fabrication of network structures}

Fabrication of network structures is possible by extending the CSA-LLM into a multistep process. After the first assembly in which the striped structure is fabricated, we rotated the substrate by $90^{\circ}$ and then carried out the second assembly, which resulted in a well-defined grid pattern, as shown in Figure 8a. The CV values of the stripe width and spacing prepared in the second assembly were comparable with those obtained in the first assembly. In contrast to the two-step CSA (without LLM) in which it is necessary to recover the hydrophilicity by plasma cleaning of the substrate with stripes before the second assembly to reproducibly obtain a grid pattern, the plasma treatment is dispensable in the two-step CSA-LLM. This can be attributed to a much shorter total assembly period of the CSA-LLM technique (tens of 
minutes) in comparison to that required for the normal CSA (tens of hours). Because of the longer total assembly period, the wettability of the substrate quickly degrades by exposure to air. It is worth noting that less wettable substrates can disturb the particle assembly. A three-step assembly with $60^{\circ}$ rotation of the substrate each time before second and third processes enables the fabrication of triangle networks, as shown in Figure $8 \mathrm{~b}$. Hence, CSA-LLM is a promising template-free technique, which can be used to fabricate various patterns.

We measured the conductivity and transmittance of a grid pattern (with a stripe width and spacing of $16 \mu \mathrm{m}$ and $81 \mu \mathrm{m}$, respectively) fabricated by CSA-LLM to evaluate the performance of the sample as a transparent conductive film. The as-prepared grid pattern was not conductive and hence, the grid pattern was treated with a plasma cleaner for 10 minutes to remove the polymers covering the gold nanoparticle surfaces. Then, the sample was calcined at $280{ }^{\circ} \mathrm{C}$ in an electric furnace for 20 minutes. The transmittance and sheet resistance were measured to be $76 \%$ and $41 \Omega / \square$, indicating that the grid pattern was both transparent and conducting. However, the sheet resistance of the grid pattern is higher than that of an ITO film with comparable transmittance. Hence, it is necessary to improve the conductivity of the grid pattern. This can possibly be achieved by increasing the stripe thickness, which is under investigation.

\section{Conclusion}

We fabricated stripe-based patterns of gold nanoparticles by CSA-LLM and investigated the relationship between the periodicity of the patterns and the experimental parameters including $t_{\mathrm{p}}$ and $l_{\mathrm{p}}$. The liquid level was manipulated by periodically aspirating the suspension out of the reservoir using a programmable syringe pump. Our results indicate that the spacing between the stripes is almost equal to the liquid-level drop distance, while the stripe volume increases 
proportionally with the deposition period. The relationship between the stripe volume and the deposition period is described by a simple equation, which is based on the mass balance taken around the meniscus tip. This equation has been demonstrated to predict the resultant stripe volume. Furthermore, we successfully fabricated grid and triangle patterns by carrying out multistep CSA-LLM. The grid pattern was transparent and conductive; however, it is necessary to increase the conductivity of the sample to allow for its application as a transparent conductive film. We are currently working on improving our technique to control the thickness of the stripe, and the results will be reported in future.

\section{Figure Caption}

Figure 1: A schematic illustration of the convective self-assembly method with liquid level manipulation

Figure 2: (a) An optical micrograph of a stripe pattern of $\mathrm{Au}$ nanoparticles. The arrow indicates the direction of the liquid-level descent. (b) An AFM height image of a stripe.

Figure 3: Optical micrographs of stripe patterns fabricated with the volume fraction $\phi=$ $1.0 \times 10^{-4}$ and different sets of operating parameters. (a) $t_{\mathrm{p}}=45 \mathrm{~s}, l_{\mathrm{p}}=133 \mu \mathrm{m}$, (b) $t_{\mathrm{p}}=30 \mathrm{~s}, l_{\mathrm{p}}$ $=267 \mu \mathrm{m},(\mathrm{c}) t_{\mathrm{p}}=7 \mathrm{~s}, l_{\mathrm{p}}=107 \mu \mathrm{m}$

Figure 4: The relation between the dropping distance of the liquid level, $l_{\mathrm{p}}$, and the stripe spacing. Error bars indicate the standard deviation of the data.

Figure 5: The relation between the deposition period $t_{\mathrm{p}}$ and the stripe width. Error bars indicate the standard deviation of the data. 
Figure 6: A schematic illustration of the vicinity of the meniscus tip.

Figure 7: The comparison between the experiments and calculation results for the dependence of the stripe volume on the deposition period. Error bars indicate the standard deviation of the data.

Figure 8: Optical micrographs of (a) grid network and (b) triangle pattern.

\section{Acknowledgement}

This work was supported in part by a Grant-in-Aid for Young Scientists (A) (No. 23686109), the Core-to-Core (CTC) Program (No. 18004) of Japan Society for the Promotion of Science (JSPS), and the Mizuho Foundation for the Promotion of Sciences.

Nomenclature

$l_{\mathrm{p}} \quad$ : liquid-level drop distance

(m)

$t_{\mathrm{p}} \quad:$ deposition period

$\phi \quad$ : volume fraction of particles

$V_{\mathrm{s}}:$ stripe volume

$\left(\mathrm{m}^{3}\right)$

$J_{\mathrm{e}} \quad$ : solvent evaporation rate at the meniscus tip

$\left(\mathrm{m}^{3} / \mathrm{m}^{2} \cdot \mathrm{s}\right)$

$L \quad$ : drying length

$W_{\mathrm{s}}:$ substrate width

\section{References}


[1] J.D. Joannopoulos, Photonics: Self-Assembly Lights Up, Nature, 414 (2001) 257-258.

[2] Y.A. Vlasov, X.-Z. Bo, J.C. Sturm, D.J. Norris, On-Chip Natural Assembly of Silicon Photonic Bandgap Crystals, Nature, 414 (2001) 289-293.

[3] S.M. Yang, H. Míguez, G.A. Ozin, Opal Circuits of Light-Planarized Microphotonic Crystal Chips, Adv. Funct. Mater., 12 (2002) 425-431.

[4] K. Lee, S.A. Asher, Photonic Crystal Chemical Sensors: pH and Ionic Strength, J. Am. Chem. Soc., 122 (2000) 9534-9537.

[5] Y. Lu, G.L. Liu, L.P. Lee, High-Density Silver Nanoparticle Film with Temperature-Controllable Interparticle Spacing for a Tunable Surface Enhanced Raman Scattering Substrate, Nano Lett., 5 (2005) 5-9.

[6] X. Tang, S.J. O’Shea, I.U. Vakarelski, Photoresist Templates for Wafer-Scale Defect-Free Evaporative Lithography, Adv. Mater., 22 (2010) 5150-5153.

[7] D. Xia, A. Biswas, D. Li, S.R.J. Brueck, Directed Self-Assembly of Silica Nanoparticles into Nanometer-Scale Patterned Surfaces Using Spin-Coating, Adv. Mater., 16 (2004) $1427-1432$.

[8] N.N. Khanh, K.B. Yoon, Facile Organization of Colloidal Particles into Large, Perfect One- and Two-Dimensional Arrays by Dry Manual Assembly on Patterned Substrates, J. Am. Chem. Soc., 131 (2009) 14228-14230.

[9] B.H. Lee, H. Shin, M.M. Sung, Patterning a Two-Dimensional Colloidal Crystal by Water-Mediated Particle Transfer Printing, Chem. Mater., 19 (2007) 5553-5556.

[10] K. Higashitani, C.E. McNamee, M. Nakayama, Formation of Large-Scale Flexible Transparent Conductive Films Using Evaporative Migration Characteristics of $\mathrm{Au}$ Nanoparticles, Langmuir, 27 (2011) 2080-2083.

[11] A.S. Dimitrov, K. Nagayama, Continuous Convective Assembling of Fine Particles into Two-Dimensional Arrays on Solid Surfaces, Langmuir, 12 (1996) 1303-1311. 
[12] S. Watanabe, K. Inukai, S. Mizuta, M.T. Miyahara, Mechanism for Stripe Pattern Formation on Hydrophilic Surfaces by Using Convective Self-Assembly, Langmuir, 25 (2009) 7287-7295.

[13] Y. Mino, S. Watanabe, M.T. Miyahara, Fabrication of Colloidal Grid Network by Two-Step Convective Self-Assembly, Langmuir, 27 (2011) 5290-5295.

[14] Y. Mino, S. Watanabe, M.T. Miyahara, Colloidal Stripe Pattern with Controlled Periodicity by Convective Self-Assembly with Liquid-Level Manipulation, ACS Appl. Mater. Interfaces, 4 (2012) 3184-3190.

[15] E. Pauliac-Vaujour, A. Stannard, C.P. Martin, M.O. Blunt, I. Notingher, P.J. Moriarty, I. Vancea, U. Thiele, Fingering Instabilities in Dewetting Nanofluids, Phys. Rev. Lett., 100 (2008) 176102. 


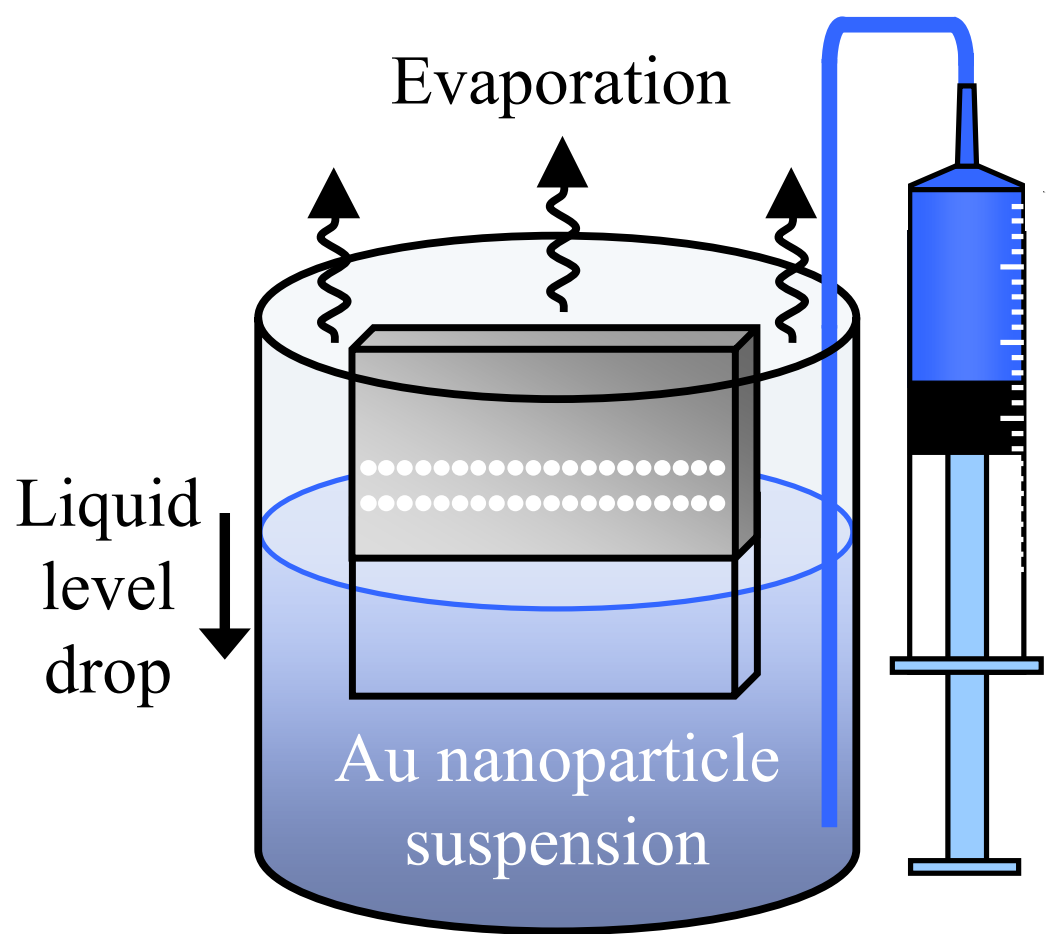

Fig.1 

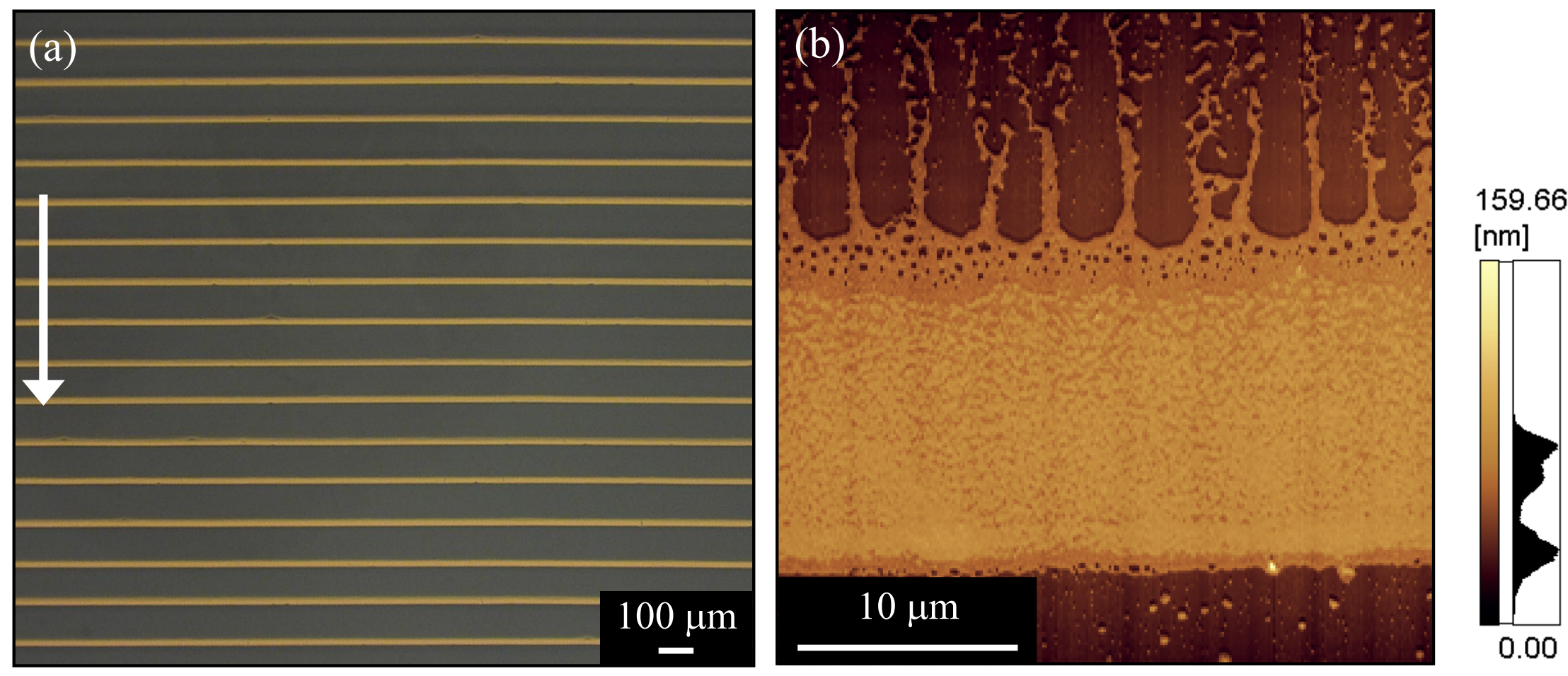

Fig.2 


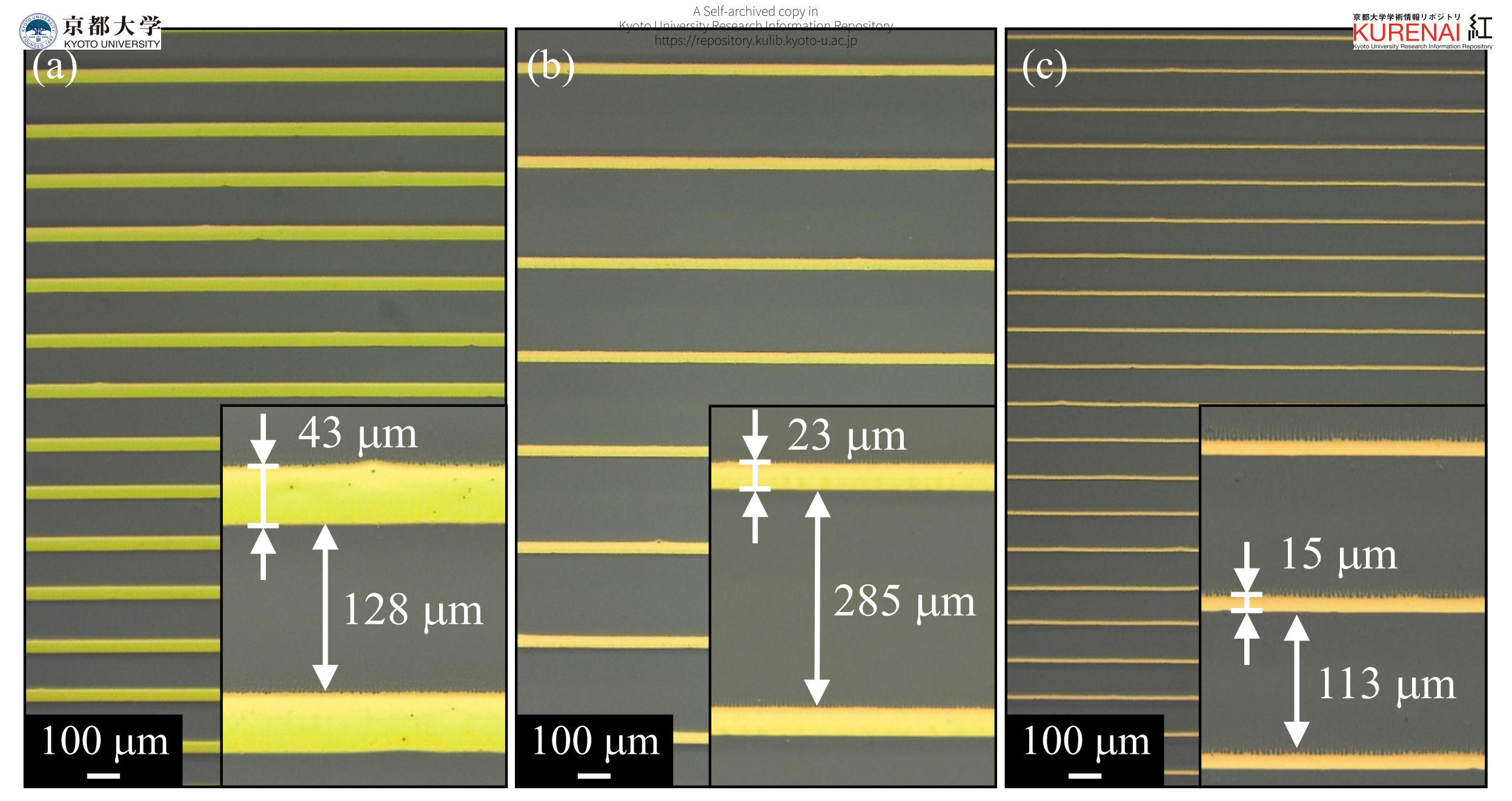

Fig.3 


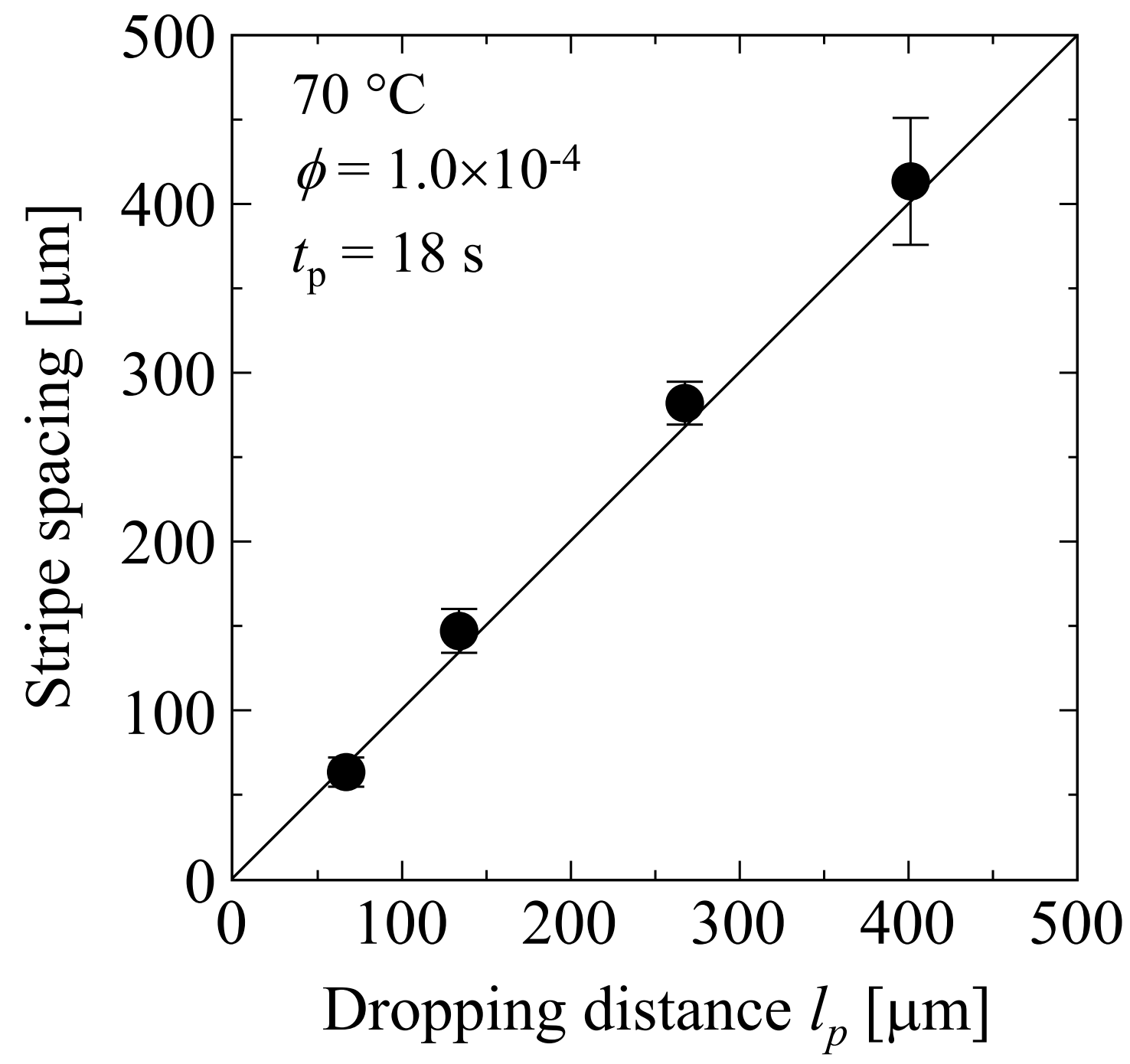

Fig.4 


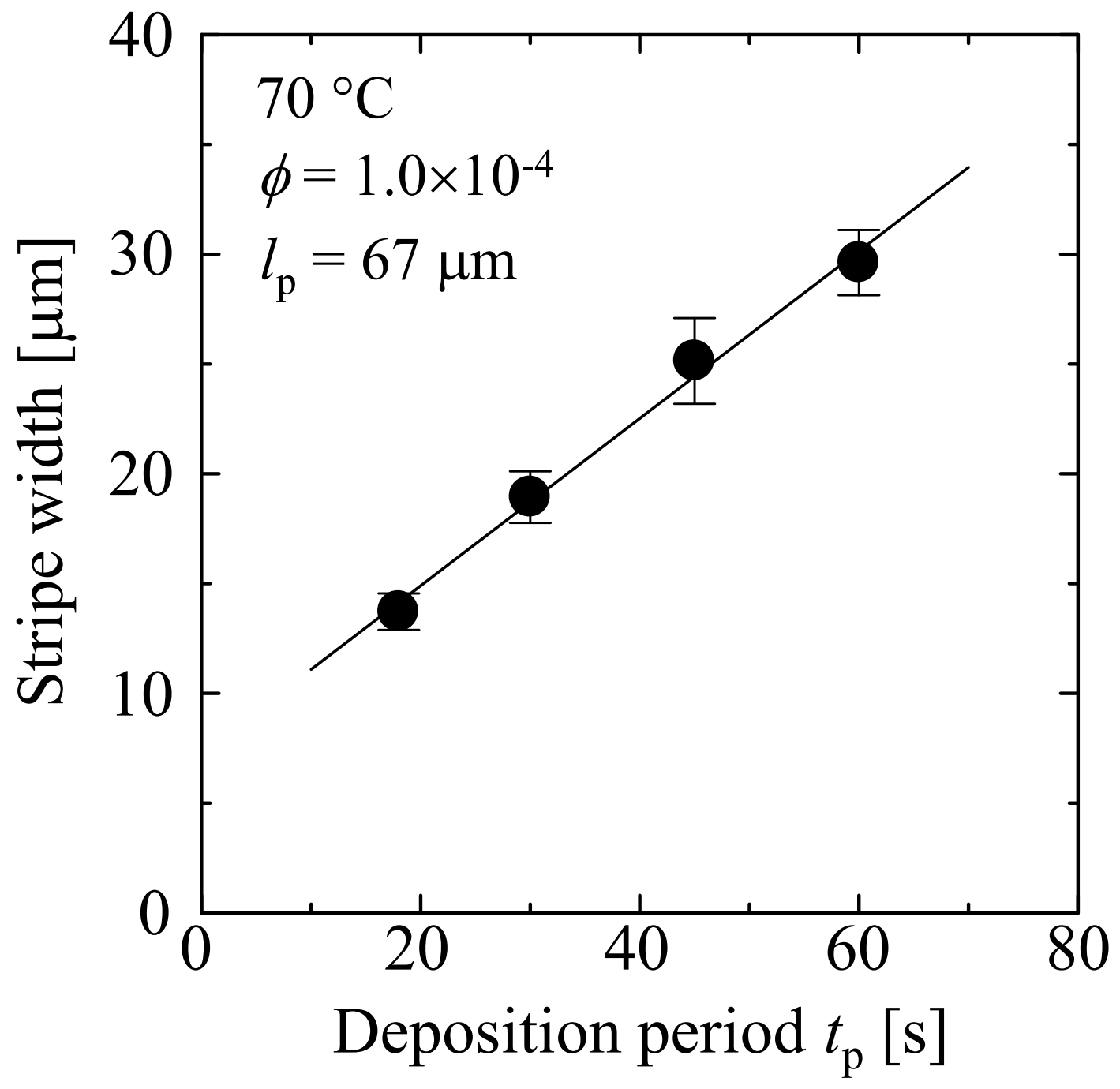

Fig.5 


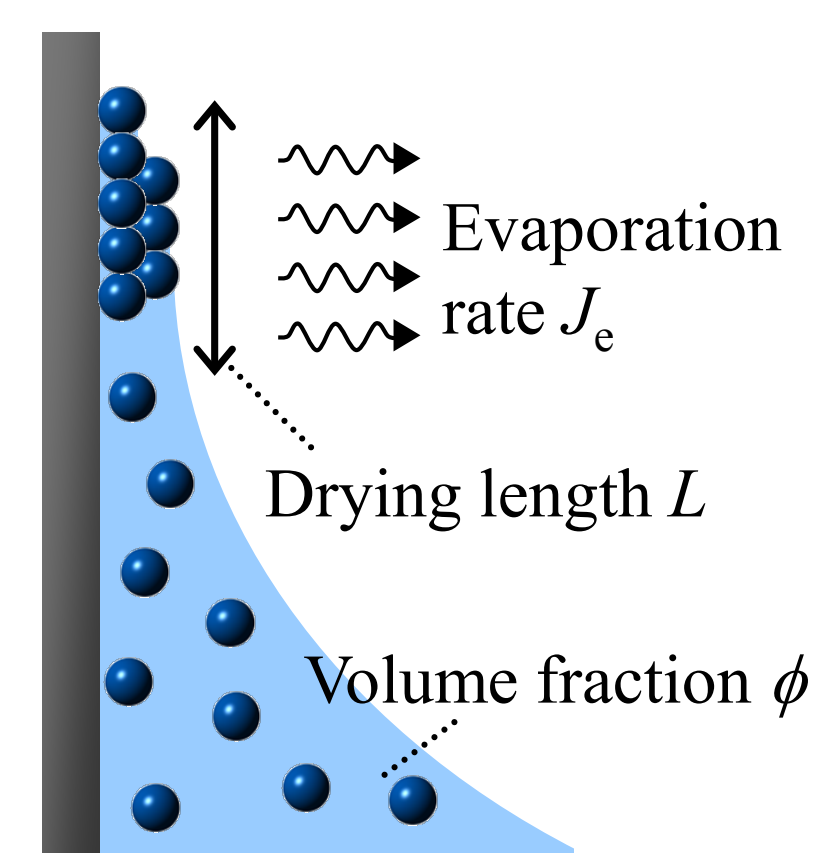

Fig.6 


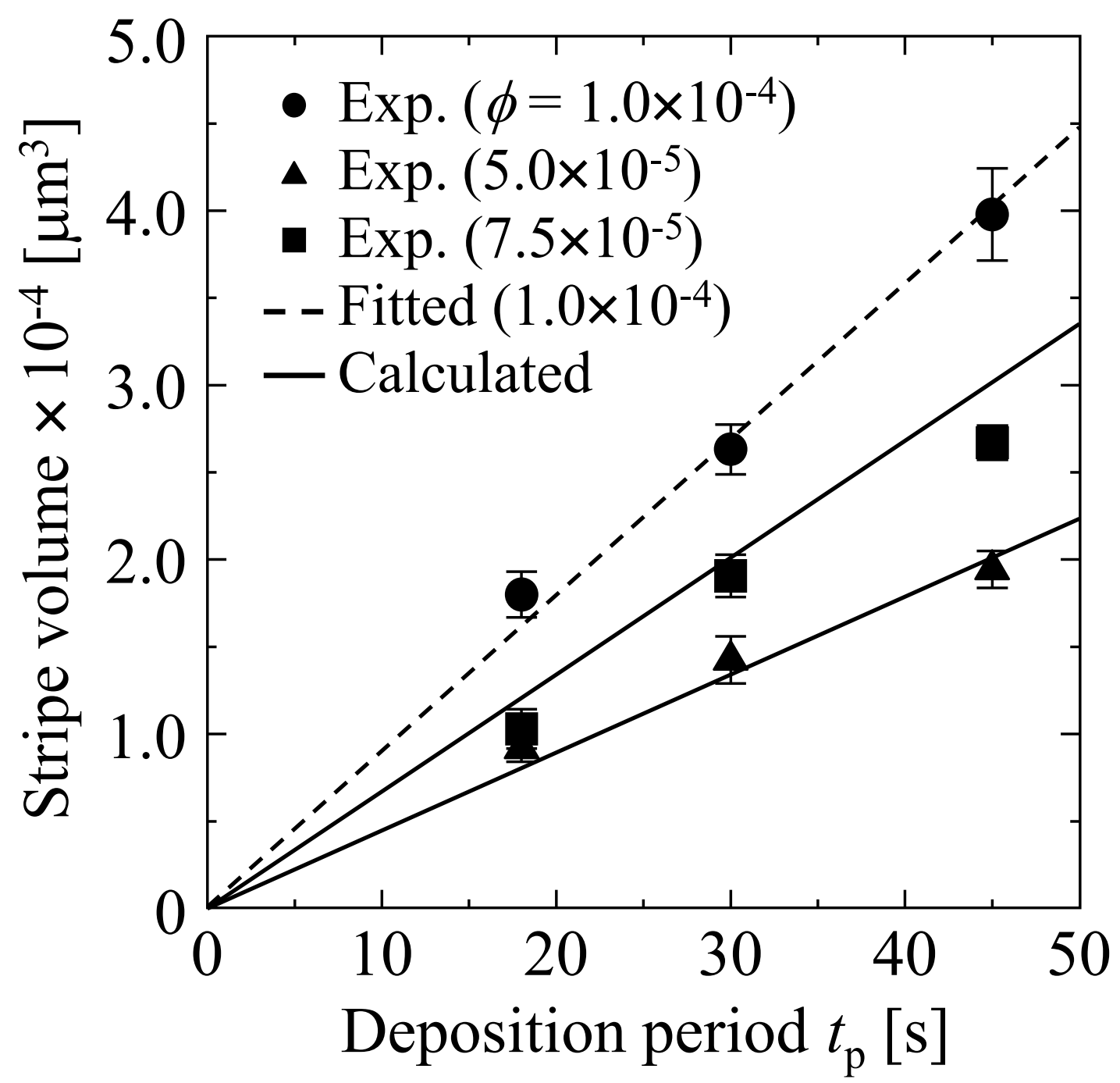

Fig.7 

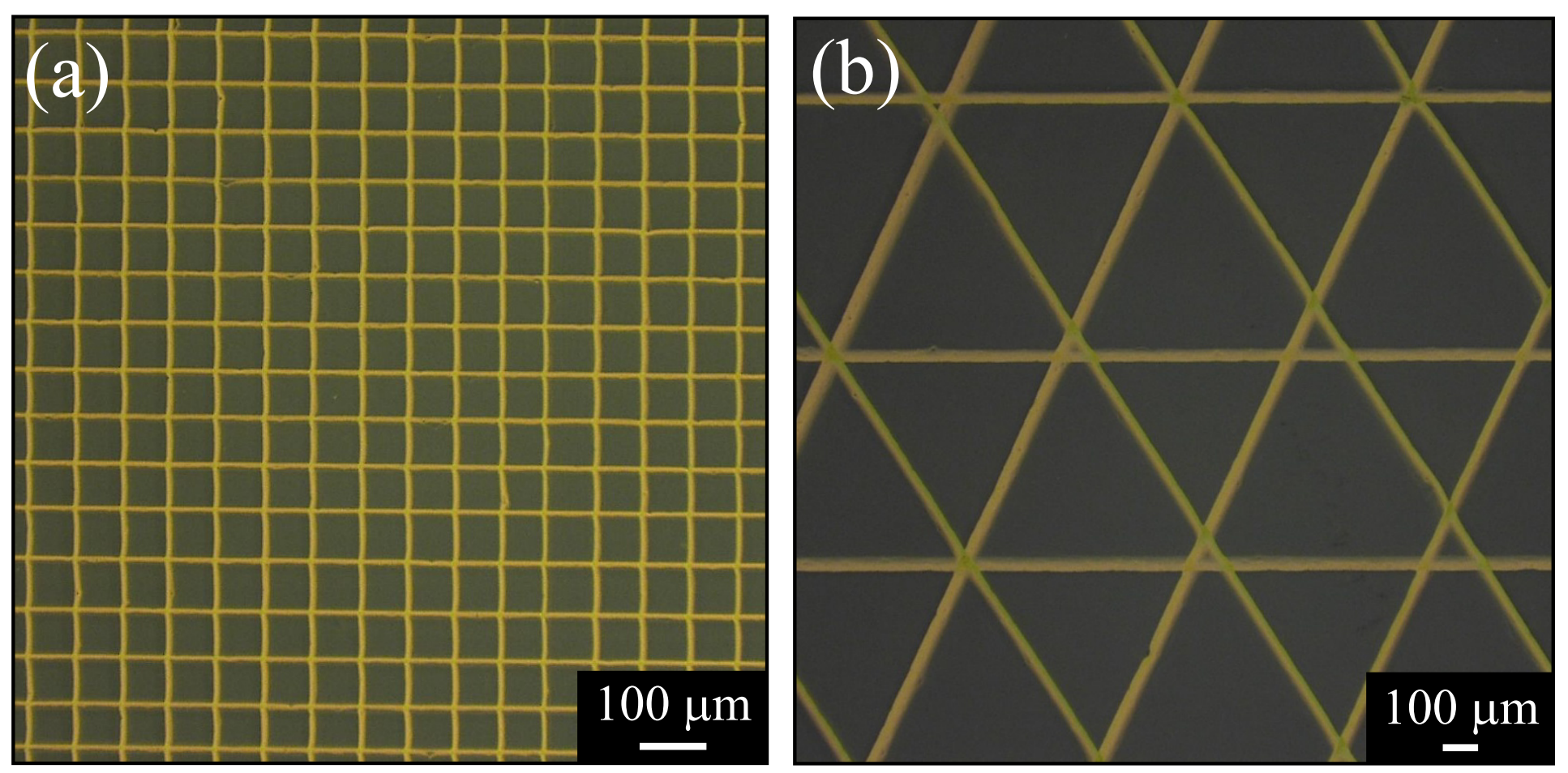

Fig. 8 